\title{
Distributed Rule-Regulated Spectrum Sharing
}

\author{
Lili Cao and Haitao Zheng * \\ Department of Computer Science \\ University of California, Santa Barbara, CA 93106 U.S.A \\ Email: \{lilicao,htzheng\}@cs.ucsb.edu
}

\begin{abstract}
Dynamic spectrum access is a promising technique to use spectrum efficiently. Without being restricted to any prefixed spectrum bands, nodes choose operating spectrum ondemand. Such flexibility, however, makes efficient and fair spectrum access in large-scale networks a great challenge. Prior work in this area focused on explicit coordination where nodes communicate with peers to modify local spectrum allocation, and may heavily stress the communication resource. In this paper, we introduce a distributed spectrum management architecture where nodes share spectrum resource fairly by making independent actions following spectrum rules. We present five spectrum rules to regulate node behavior and maximize system fairness and spectrum utilization, and analyze the associated complexity and overhead. We show analytically and experimentally that the proposed rule-based approach achieves similar performance with the explicit coordination approach, while significantly reducing communication cost.
\end{abstract}

Index Terms-Cognitive radio, spectrum access, rule-based, distributed, poverty line, fairness.

\section{INTRODUCTION}

Wireless innovation and deployment has been stifled by the shortage of radio spectrum [13]. The shortage problem comes from the current (and historical) spectrum management policies that assign spectrum bands statically to wireless technologies in long-term licenses. While utilization of assigned spectrum can be as low as 5-10\% [13], new technologies in search of market availability such as WiFi are forced to utilize unlicensed spectrum bands, resulting in crowded spectrum utilization and unpredictable performance from aggressive deployments [2].

The ideal and necessary solution to the artificial shortage problem is dynamic spectrum access. In this new model, future wireless devices no longer operate on statically assigned spectrum, but acquire spectrum on-demand. Using cognitive radios [8], [15], devices opportunistically utilize unused spectrum without disrupting operations of legacy spectrum owners, e.g. TV broadcasts. Peers share locally available spectrum to maximize spectrum utilization.

One key challenge in dynamic spectrum access networks is how to maintain efficient spectrum sharing among peers. While maximizing spectrum utilization is the primary goal, we also need good sharing mechanisms to provide fairness across wireless nodes. A node seizing spectrum without coordinating with others can cause harmful interference to neighbors and hence reduce spectrum utilization.

Corresponding author. Manuscript received Mar. 2007, revised Sept. 2007. Part of this work was published at IEEE DySPAN conference, November 2005.
In this paper, we consider the problem of distributed collaborative sharing where a group of wireless nodes agree to share available spectrum and maximize a predefined system utility. This maps to scenarios where a single service provider deploys a large number of wireless nodes and enforces collaboration agreements among nodes. For example, future WiMAX and/or $\mathrm{WiFi}$ access points share spectrum to provide city-wide wireless coverage [1]. Equipped with cognitive radios, these future access points can utilize multiple non-consecutive channels concurrently to offer high-speed wireless access. Assuming nodes can sense and discover locally-available spectrum, we reduce the problem of spectrum sharing into a constrained channel allocation problem. We seek distributed solutions that efficiently and fairly distribute spectrum channels to nodes such that any conflicting nodes are assigned with orthogonal channels. We model the requirement of fairness using the proportional fairness [20] based system utility.

In this context, prior work [6] proposed a distributed algorithm using explicit communication. In this approach, nodes self-organize into coordination groups on-demand and adjust spectrum allocation within each group to improve local system utility. By regulating coordination procedures, this approach converges quickly and produces spectrum allocations that closely approximate the optimal solution. However, this approach also requires nodes to communicate and exchange coordination message frequently. This explicit coordination model requires real-time reliable communication among nodes, which is not always available.

In this work, we propose an alternative distributed, collaborative spectrum sharing approach with minimum coordination overhead. Instead of exchanging coordination messages, nodes act independently based on their local observation of spectrum condition. Nodes coordinate implicitly and regulate their actions by complying with a set of predefined rules [27]. In this case, while nodes act independently and tend to prioritize their own performance, their compliance with the rules promotes efficient and fair spectrum sharing. Compared to approaches with explicit coordination, this approach greatly simplifies implementation and significantly reduces coordination overhead.

This paper makes four contributions:

- First, we design five rules that regulate nodes' spectrum access to tradeoff fairness and utilization with communication costs and algorithm complexity.

- Second, we prove analytically that a system under these rules converges in finite iterations, and that each node has a guaranteed amount of spectrum allocation.

- Third, we design detailed algorithms to implement these 


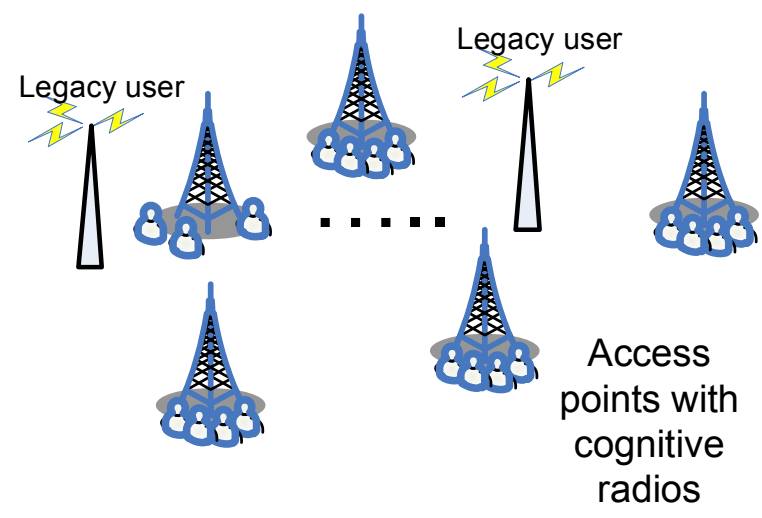

Fig. 1. An illustrative scenario of distributed spectrum sharing. Access points identify locally available channels that will not disrupt the operations of legacy spectrum owners such as TV broadcasts. They also share these available spectrum with peers, and access spectrum to connect their subscribers.

rules and discuss practical issues.

- Finally, we use extensive simulations to quantify the impact of rules on spectrum access, while comparing implicit and explicit coordination approaches in terms of efficiency and complexity. We also examine the impact of network topologies using both synthetic and real traces.

The rest of the paper is organized as follows. We begin in Section II with background on spectrum allocation and existing solutions. In Section III we describe the spectrum allocation problem. Next in Section IV, we present the rulebased spectrum allocation system and develop different spectrum rules. We evaluate the system performance and complexity analytically in Section V. We then provide detailed implementations of the proposed rules in Section VI. Next in Section VII, we conduct experiments to evaluate the proposed coordination based approaches. We discuss practical issues in VIII and conclude in Section IX.

\section{BACKGROUND AND RELATED WORK}

In this section we introduce dynamic spectrum access systems, its key challenges and existing work. Figure 1 illustrates a motivating scenario where a number of WiMAX/WiFi access points share spectrum to connect their subscribers. These access points, hereby referred to as nodes, are equipped with cognitive radios. They sense spectrum to identify usable channels without affecting operations of legacy spectrum users, such as TV broadcasts. For a given set of available channels, the spectrum allocation problem is to allocate channels to access points so that conflicting access points are assigned with different channels. The optimal allocation maximizes a predefined system utility, e.g. proportional fairness or total spectrum utilization.

\section{A. Challenges}

Solving the problem of spectrum allocation faces the following key challenges:

- NP-hard optimization under non-linear interference constraints: The optimization problem is subject to a set of interference constraints. Nodes in close proximity interfere with each other and can not use the same spectrum band while well-separated nodes can reuse the same spectrum. In general, the optimal spectrum allocation problem is known to be NP-hard [20]. In this paper, we model the interference constraints using the widely-used protocol interference model [11] and represent interference constraints as a conflict graph. In Section VIII, we also discuss practical considerations on how to improve this model for more realistic characterization of interference.

- Fast system convergence: To support dynamic spectrum access, nodes need to exercise an efficient allocation algorithm to allocate resource in real-time, with only local view of the network. In a distributed approach where nodes independently adjust their spectrum usage, the system must converge quickly and maintain stability.

\section{B. Related Work on Spectrum Allocation}

There are multiple complementary approaches to allocate spectrum, each designed for different scenarios. These include centralized allocation [23], [20], distributed game-theory driven sharing [17], [19], [24] and distributed collaborative sharing [6], [27].

In the centralized approach, a central server collects network information and allocates channels to nodes to maximize a predefined system utility. The work in [4], [10], [21] proposed the use of central spectrum server model, and developed several heuristics based centralized approximations for a limited number of users. The work in [20], [28] shows that the heuristic based centralized algorithms perform similarly to the global optimum. While well-suited for small-scale networks, this approach suffers from lack of scalability for large-scale networks.

In the game-theory driven approach, nodes compete to maximize self benefits, e.g. competing wireless service providers access spectrum to maximize their service quality or revenue [10], [8], [18], [19], [21]. In this case, nodes act against each other to maximize their own utility. These distributed approaches are well-suited for scenarios where nodes are selfish and compete with each other.

In the distributed collaborative sharing, nodes collaborate to maximize a predefined system utility. Our prior work [6] proposes a distributed collaborative approach using explicit coordination and communication. In this case, nodes selforganize coordination groups, exchange control messages, and determine the optimal spectrum allocation in each group to maximize the system utility. This approach is well-suited for scenarios where nodes are controlled by a single entity who can enforce a cooperation agreement among all the nodes [14], [22].

In this paper, we focus on distributed collaborative approaches. However, our work differs from the explicit coordination approach [6] because it does not require nodes to communicate directly and negotiate spectrum allocations. Instead, nodes coordinate implicitly - they adjust self channel usage independently based on rules. By regulating node behaviors using predefined rules, we show that this implicit 
approach performs closely to that of the explicit approach while significantly reducing coordination overhead.

\section{Spectrum Allocation Problem}

In this section, we describe the problem of spectrum allocation using the scenario of WiMAX/WLAN access points. We start with notations and assumptions of the network model, then define the channel allocation problem.

\section{A. Assumptions}

As shown in Figure 1, we assume a network of $N$ nodes (access points) indexed from 0 to $N-1$, each accessing spectrum to connect their subscribers, whose traffic is backlogged. We assume that the spectrum is divided into $M$ nonoverlapping orthogonal channels indexed 0 to $M-1$. Equipped with cognitive radios, each node can communicate through multiple non-consecutive channels. We assume that each node transmits using a predefined combination of radio parameters (e.g. power and modulation), and focus on achieve conflictfree spectrum allocation by assigning conflicting nodes with orthogonal channels ${ }^{1}$.

We assume nodes can reliably identify nearby legacy spectrum owners, and opportunistically utilize locally unused spectrum. In our model, each node observes a set of available channels (defined by a channel availability matrix) that it can use without disrupting the operations of legacy spectrum owners.

We assume nodes can reliably identify conflicting peers using interference detection techniques [22], [14]. We model the interference among nodes using the commonly-used protocol model [11]. It models the interference condition as a pair-wise binary matrix - any two nodes either conflict and can not use the same channel simultaneously, or they do not conflict and can reuse the same channel. While this is a simplified abstraction of the complex interference conditions, in practice, it makes the optimization problem tractable. In Section VIII we discuss extensions to more complex interference models.

Finally, we address the spectrum allocation problem from a MAC layer perspective. Because nodes (access points) are static, we assume a pseudo-static interference environment without considering the impact of fast-scale channel fading. In addition, we assume channels are homogeneous, i.e. they offer the same bandwidth and have similar interference characteristics. This assumption is well-suited for scenarios where nodes share a set of channels within the same carrier. In the following, we assume that the channels offer an average bandwidth of 1 , and the interference condition is uniform across all the channels. In Section VIII we also discuss extensions to scenarios with heterogeneous channels.

\section{B. System Model}

We introduce the following notations to model the system:

\footnotetext{
${ }^{1}$ While the proposed approach can be integrated with other parametertuning techniques such as power control, we will defer this investigation to a later study.
}

- Channel availability - Let $\mathbb{L}=\left\{l_{m, n} \mid l_{m, n} \in\right.$ $\{0,1\}\}_{M \times N}$, a $M$ by $N$ binary matrix, represent the channel availability:

$$
l_{m, n}= \begin{cases}1, & \text { channel } m \text { available at node } n \\ 0, & \text { channel } m \text { occupied by a legacy user. }\end{cases}
$$

Let $L(n)=\sum_{m=0}^{M-1} l_{m, n}$ represent the total number of channels available at node $n$.

- Interference constraint - Let $\mathbb{C}=\left\{c_{n, k} \mid c_{n, k} \in\right.$ $\{0,1\}\}_{N \times N}$, a $N$ by $N$ binary matrix, represent the interference constraints among nodes:

$c_{n, k}= \begin{cases}1, & \text { node } n \text { and } k \text { conflict with each other } \\ 0, & \text { node } n \text { and } k \text { can reuse the same channel. }\end{cases}$

Define the "neighbor" of node $n$ as any node $k$ with $c_{n, k}=1$, and $d(n)=\sum_{k=0}^{N-1} c_{n, k}$ as the total number of conflicting neighbors of $n$.

- Conflict-free channel assignment - Let $\mathbb{A}=$ $\left\{a_{m, n} \mid a_{m, n} \in\{0,1\}\right\}_{M \times N}$, a $M$ by $N$ binary matrix, represent a spectrum allocation:

$$
a_{m, n}= \begin{cases}1, & \text { channel } m \text { is assigned to node } n \\ 0, & \text { otherwise. }\end{cases}
$$

In addition, $\mathbb{A}$ satisfies all the constraints defined by $\mathbb{C}$, that is,

$$
a_{m, n} \cdot a_{m, k}=0, \text { if } c_{n, k}=1, \forall n, k<N, m<M \text {. }
$$

\section{The Optimization Problem}

The problem of spectrum allocation is modeled by the following constrained optimization problem:

$$
\mathbb{A}^{*}=\underset{\mathbb{A}}{\operatorname{argmax}} U(\mathbb{A}),
$$

subject to Interference constraints defined by (1).

$U($.$) represents the system utility function. In this paper, we$ focus on the scenario where APs belong to a single entity such as an enterprise [14], [22]. The spectrum allocation is not driven by monetary gain but system-wide fairness. We use proportional fairness [16], [20], which is regarded as a compromise between max-min fairness and maximum throughput scheduling [12]:

$$
U_{\text {fairness }}(\mathbb{A})=\sum_{n=0}^{N-1} \log R_{\mathbb{A}}(n)=\sum_{n=0}^{N-1} \log \sum_{m=0}^{M-1} a_{m, n} \cdot l_{m, n}
$$

where $R_{\mathbb{A}}(n)=\sum_{m=0}^{M-1} a_{m, n} \cdot l_{m, n}$. represents the throughput that node $n$ gets under assignment $\mathbb{A}$.

Because of the interference constraint and the non-linear optimization function, this optimization problem is known to be NP-hard [20]. In this paper, we focus on heuristic based distributed solutions. In the following section, we discuss the proposed rule-regulated spectrum allocation approach where nodes adjust their spectrum usage $\mathbb{A}$ to maximize the system utility $U_{\text {fairness }}$ while subjecting to the interference constraints. 


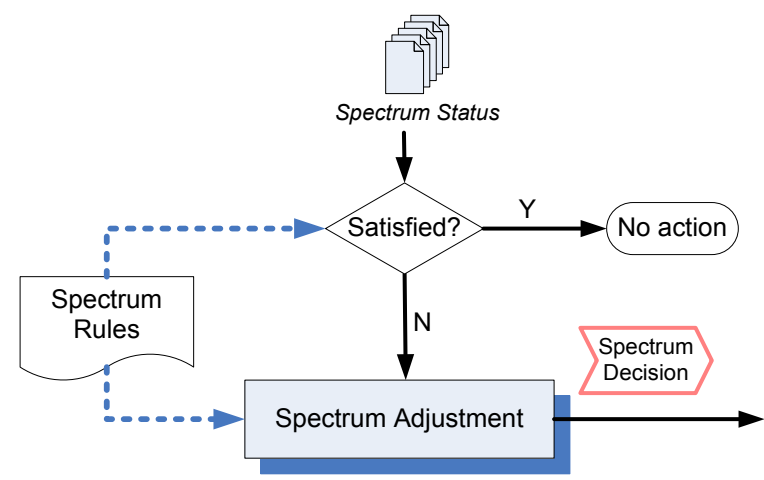

Fig. 2. The proposed rule-regulated spectrum allocation. Each node observes spectrum usage status and identifies whether to adjust spectrum usage. If so, they adjust based on a predefined spectrum rule.

\section{Rule-Regulated Spectrum Sharing}

In the proposed system, nodes observe local conditions and neighbors' actions and independently adapt their spectrum usage. Their behavior is regulated by a set of rules defined by spectrum regulators. In contrast to the explicit coordination approach [6], nodes tend to prioritize their own performance with minimal regard to system utility. However, their compliance with the rules promotes efficient and fair spectrum sharing. In the following, we start by describing the high-level concept, and then discuss the detailed rule design.

\section{A. System Overview}

Figure 2 illustrates the operation at each node to select channels. Each node $n$ performs spectrum sensing to identify its spectrum usage, i.e. $R_{\mathbb{A}}(n)$. Using spectrum rules, each node checks whether it needs to update its channel selections. If an update is required, nodes rely on rules to determine the appropriate channels to use.

The key challenge in this design is how to define the spectrum rules. The rules specify how many and which channels a node should use, such that fairness and utilization can be achieved. The estimation should not be overly aggressive and bring excessive contention, or overly conservative and result in spectrum under-utilization. Further, in a distributed system, each node can only act based on limited local view of the system.

We design rules based on the observation from the collaborative spectrum sharing system with explicit coordination [6]. In this prior work, we have shown that when the system converges, the number of channels a node obtains is low-bounded. This lower bound, defined as "poverty line," represents the minimum amount of spectrum a node is entitled to. If a node $n$ has $L(n)$ available channels and $d(n)$ conflicting neighbors, its poverty line is

$$
P L(n)=\left\lfloor\frac{L(n)}{d(n)+1}\right\rfloor .
$$

Results in [6] also show that the poverty line is very tight, making it a relatively accurate estimation of the number of channels a node should get in order to maximize fairness. In this paper, we propose to use this measure as the basis of spectrum rules.

Next, we propose five different rules that tradeoff between performance and signaling complexity for different application scenarios.

\section{B. Rules for Conflict Free Channel Assignment}

We begin by describing rules that lead to conflict free spectrum usage. Conflict free channel usage is one of the scheduling methods that allow for explicit and guaranteed throughput provisioning and control over packet delay. In this case, nodes always select idle channels, i.e. channels unclaimed by conflicting peers. To provide fairness, the rules limit the number of channels each node can access.

Rule A (Uniform Idle Preference): Each node adjusts its spectrum usage to $\Omega=\min _{n} P L(n)$ number of idle channels.

Service providers can optimize the value of $\Omega$ for the entire network. However, nodes experiencing intensive interference from legacy nodes (small $L(n)$ ) or other peers in a crowded area (large $d(n)$ ) can limit the the value of $\Omega$, leading to less than ideal spectrum utilization. Therefore, adapting $\Omega$ to each node's interference condition is preferred. Next we propose a rule which gives each node $n$ exactly $P L(n)$ number of channels.

Rule B (Poverty Exact Idle Preference): A node $n$ selects exactly $P L(n)=\left\lfloor\frac{L(n)}{d(n)+1}\right\rfloor$ idle channels. If the number of idle channels $<P L(n)$, it "seizes" channels from "richer" nodes without affecting "poor" nodes. A node conflicting with a "poor" node will sense the conflict and give up the channel and switch to other channels following the same procedure.

To $n$, a neighbor is "richer" if it uses more channels than $n$; otherwise it is "poor". Rule B requires that each node has knowledge of the number of neighbors $d(n)$, and the channel selection of each neighbor in order to identify "richer" nodes. To "grab" non-idle channels, a node $n$ marks the channels occupied by "poor" neighbors as busy, and the rest as idle. node $n$ then selects a set of channels from the "idle" channels until its channel occupancy reaches $P L(n)$. The efficiency of grabbing depends on the set of channels selected. In Section VI, we present the detailed procedure.

A limitation of Rule B is that each node only attempts to use $P L(n)$ channels. Since $P L(n)$ represents a lower bound on spectrum usage derived using a collaboration based approach [6], Rule B could under-utilize available spectrum. Therefore, we devise an improved rule that guarantees the poverty line for each node while letting some nodes go beyond their poverty lines.

Rule C (Poverty Guided Idle Preference): A node $n$ selects channels from idle channels. Only if there are not enough idle channels to reach $P L(n)$ does node n "grab" channels from "richer" neighbors. The number of channels it can grab from any "richer" node $r$, is $\max \{0, \min \{C(r)-P L(n), P L(n)-$ $C(n)\}\}$ where $C(n)$ and $C(r)$ are the current spectrum usage of node $n$ and $r$.

Rule $\mathrm{C}$ allows nodes who have attained their poverty line to seize additional idle channels. It still allows nodes below 
their poverty line to take channels from "richer" neighbors. However, each grabbing can not reduce a "richer" node's spectrum below the grabber's poverty line, avoiding cycles of nodes grabbing channels from each other in turn. In particular, a node $n$ can collect all the channels used by its "richer" neighbors but not "poor" neighbors into a channel pool, reserve $P L(n)$ channels for each "richer" neighbor and "grab" from the rest of the pool. Similarly, the channel selection procedure impacts the efficiency of the grabbing, which will be described in Section VI. We note that Rule C does not require each node to have knowledge of its neighbors' poverty line.

We note that the performance of conflict free channel assignments such as Rules B and C depends on the granularity of spectrum partition, i.e. the number of channels $M$. When $M$ is small compared to the number of neighbors $d(n)$, some nodes may have a poverty line of zero, and hence no performance guarantee. In this case, the system can increase granularity by partitioning time, e.g. a channel is defined as a frequency band at a particular time slot.

\section{Rules for Contention-based Channel Assignment}

Broadcasting spectrum usage to neighbors might be undesirable for a number of reasons, including privacy concerns and protection against jamming from malicious nodes. For these reasons, we now describe several rules that do not require knowledge of neighbors' spectrum usage.

In this approach, on each channel, nodes follow a set of random access rules such as CSMA to compete fairly for channel access and avoid conflict. Each node performs contention detection, i.e. listens to the channel before initiating any transmission. It initiates the transmission only when the channel is idle for some given time. Otherwise, it backs off and delays the action for a short period. Because channels have different contention conditions, nodes should invoke independent contention detection and backoff process on each channel. The penalty of such random access is the overhead of contention detection even if there is only one node on the channel.

The rules specify the number of channels nodes should use and how to select these channels. We propose the following two rules depending on whether nodes have information about their poverty line.

Rule D (Selfish Spectrum Contention): Each node $n$ can use up to the $\Psi$ channels providing the highest throughput. Communication on each channel is through CSMA based time contention.

We note that the poverty line concept can provide a reference for choosing different value of $\Psi$ for different nodes. Since the poverty line represents throughput attainable from conflict free spectrum usage, $\Psi_{n}$ should be larger than $P L(n)$ to account for channel contention. Note that in the random access scenario, $P L(n)$ can still be computed using only the number of neighbors, which can be estimated by listening to MAC control packets.

Rule E (Poverty Guided Selfish Spectrum Contention): This rule is the same as Rule D except the number of channels each node $n$ can use is limited by $\Psi_{n}=\max (\alpha \cdot P L(n), 1), \alpha \geq 1$.
Both rules encourage nodes to act selfishly. Nodes monitor channel conditions and switch to channels that provide the best throughput, even if such a switch might reduce performance for other neighbors. One question is how to choose the best channels with maximum capacity and minimum contention. For the purpose of illustration, we use the number of competing nodes as an indicator of channel quality. Hence, following Rule D or E, nodes always switch to channels with the least number of competing nodes. This also makes both rules efficient.

\section{AnAlytical Bounds}

In this section, we derive analytical bounds on the performance and complexity of the proposed rules.

\section{A. Conflict-Free Rules}

First, it is straightforward to show that Rule A provides a conflict-free spectrum allocation.

Theorem 1: Rule A guarantees a conflict free spectrum allocation. (Proof in appendix A).

Next, we illustrate the performance and complexity bounds of Rules B and C. Assuming no two neighbors modify their spectrum/channel usage simultaneously, the system will reach equilibrium after a finite number of iterations. Equilibrium is the state where nodes have no incentive to adjust their spectrum usage.

Theorem 2: Using Rule $B$ or $C$, the system reaches an equilibrium after an expected number of at most $O\left(N^{2}\right)$ node spectrum modifications. In equilibrium, there is no conflict in spectrum usage, and each node's spectrum usage is no less than its Poverty Line $P L(n)$ (equal to $P L(n)$ for Rule $B$ ). (Proof in appendix B).

\section{B. Contention-based Rules}

Theorem 3: Using Rule $D$ or E, the system will reach an equilibrium after at most $\Lambda \times M$ node spectrum modifications. $\Lambda$ is bounded by $O\left(N^{2}\right)$. (Proof in appendix $\mathrm{C}$ ).

The choice of $\Psi$ and $\alpha$ depends on specific random access mechanisms. To analyze their impact, we use a simple model to characterize channel sharing. A node contending with $m$ other nodes on a channel gets $1 /(\lambda \cdot(m+1))$ of the channel throughput, where $\lambda$ is the contention penalty. We refer to this model as the $(\lambda, m)$ model. When $\lambda=1.8$, this model matches the experimental test and analytical results for CSMA-based IEEE 802.11 b systems at $11 \mathrm{Mbps}$ in [9].

We show that Rule D guarantees a lower bound on node throughput when $|L(n)|=M$ (See Theorem 4). The bound is only tight when $\Psi=M$, however, and cannot be used to derive the optimal value of $\Psi$. Theoretical analysis of Rules $\mathrm{D}$ and $\mathrm{E}$ is ongoing work.

Theorem 4: Using Rule $D$ and $(\lambda, m)$ model, a node $n$ 's throughput is lower-bounded by $L B(n, \Psi)=$ $\frac{1}{\lambda\left\{\left\lfloor\frac{d(n)}{M}\right\rfloor+1\right\}},(\Psi=1) ; \frac{\Psi}{\lambda\left\{\left\lfloor\frac{\Psi \times d(n)}{M}\right\rfloor+2\right\}}, \quad(1<\Psi<M) ;$
$\frac{M}{\lambda\{d(n)+1\}},(\Psi=M) .($ Proof in appendix D) 
TABLE I

REQUIRED INFORMATION TO EXECUTE THE RULES

\begin{tabular}{|c|c|c|c|c|}
\hline & $\begin{array}{c}\text { channel } \\
\text { idle/busy }\end{array}$ & $\begin{array}{c}\text { No. neighbors } \\
d_{n}\end{array}$ & $\begin{array}{c}\text { each neighbor's } \\
\text { channel usage }\end{array}$ & $\begin{array}{c}\text { channel } \\
\text { utilization }\end{array}$ \\
\hline Rule A & $\checkmark$ & & & \\
\hline Rule B & $\checkmark$ & $\checkmark$ & $\checkmark$ & \\
\hline Rule C & $\checkmark$ & $\checkmark$ & $\checkmark$ & \\
\hline Rule D & $\checkmark$ & & & $\checkmark$ \\
\hline Rule E & $\checkmark$ & $\checkmark$ & & $\checkmark$ \\
\hline
\end{tabular}

\section{RULE IMPLEMENTATIONS}

In this section, we provide implementation details on how each node applies the proposed rules to select channels. Nodes execute these procedures after detecting a conflict or that its actual usage is less than its poverty line. Table I summarizes the information required at each node to execute each of the five rules. They provide an intuitive measure of each rule's required communication complexity.

\section{A. Implementing Rule $B$ and $C$}

We start with Rule B and C that lead to conflict free channel assignment. Implementation of Rule $\mathrm{B}$ and $\mathrm{C}$ requires the following two mechanisms:

\section{- Neighbor Spectrum Usage Discovery.}

Rule B and C require knowledge of neighbors' spectrum usage. In particular, nodes (especially those below their poverty line) need to know the set of channels each neighbor currently occupies. This can be done by each node broadcasting their channel usage embedded in beacon broadcasts [26]. These broadcasts are simple to implement, and their corresponding overhead is much smaller compared to that of explicit coordination based approaches [6], [20]. An alternative is to use a sophisticated spectrum analyzer.

- Isolated Adjustment.

Rule B and C assume no simultaneous spectrum adjustments by neighboring nodes. Since nodes can independently update their spectrum usage, a conflict occurs if two neighbors simultaneously switch to the same idle channel. Therefore, after a node decides to switch to a new channel, it computes a short random wait time before starting a transmission. If it detects activity on the channel during the wait time, it marks the channel as busy, and cancels the channel switch.

Using the conflict discovery module, each node $n$ derives $d(n)$, $L(n)$ and poverty line $P L(n)$. If $n$ 's channel usage fells below $P L(n)$ or upon observing a conflict, it adjusts spectrum usage in two phases.

\section{- Phase I: Channel Classification.}

In this phase, node $n$ classifies its available channels into three disjoint sets: reserved set $R$, conflicting set $O$, and idle set $I$, defined in Table II. The reserved channel set includes all the channels that are not eligible for $n$, preventing $n$ from grabbing channels from "poor" neighbors or over-grabbing from "richer" neighbors. The conflicting set and idle set identify all the channels eligible for $n$ to grab. The channels in the idle set have the highest priority since the corresponding "grabbing" does
TABLE II

CHARACTERIZATION OF CHANNELS

\begin{tabular}{|c|c|l|l|}
\hline Set & Name & Definition (Rule B) & Definition (Rule C) \\
\hline$R$ & Reserved & $\begin{array}{l}\text { Channels occupied } \\
\text { by poor neighbors } \\
\text { of } n\end{array}$ & $\begin{array}{l}\text { channels occupied by } \\
\text { poor neighbors of } n \text { and } \\
\text { the } P L(n) \text { smallest- } \\
\text { ID channels of each } \\
\text { "richer" neighbor of } n\end{array}$ \\
\hline$O$ & Conflicting & $\begin{array}{l}\text { Channels occupied by neighbors of } n \text { but } \\
\text { not in set } R\end{array}$ \\
\hline$I$ & Idle & $\begin{array}{l}\text { Channels not occupied by any neighbor of } \\
n\end{array}$ \\
\hline
\end{tabular}

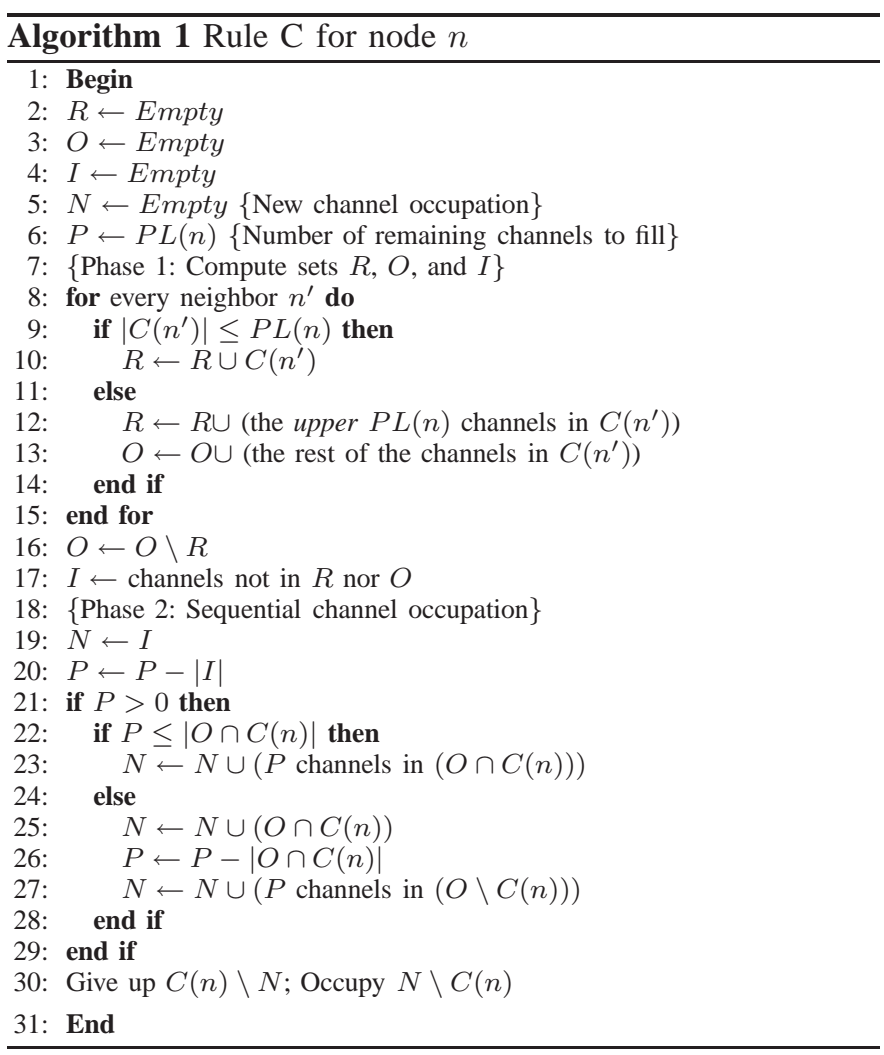

not disturb any neighbor. It should be noted that before spectrum modification, the node $n$ 's channel usage $C(n)$ may intersect with $R, O$, and $I$; the cardinality of $C(n)$ could be below or above $P L(n)$.

- Phase II: Channel Selection.

In this phase, node $n$ selects channels to use from sets $I$ and $O$. It always starts from the set $I$ to avoid disturbing neighbors. Only if $|I|<P L(n)$ will it select from the conflicting set $O$. When selecting channels from a channel set $(I$ or $O$ ), node $n$ should select from its current channel occupation with higher priority, reducing disturbance to neighbors and avoiding meaningless channel usage modifications. For Rule B, the node sequentially chooses $P L(n)$ channels from sets $I \cap C(n), I \backslash C(n)$, $O \cap C(n)$, and $O \backslash C(n)$. For Rule $\mathrm{C}$, the node seizes all channels in $I$, and if it hasn't reached $P L(n)$, then it sequentially chooses its channel usage from $O \cap C(n)$, and $O \backslash C(n)$. During the channel selection, a node always starts from the smallest channel IDs in a channel set. We list the detailed procedure in Algorithms 1 and 2. 


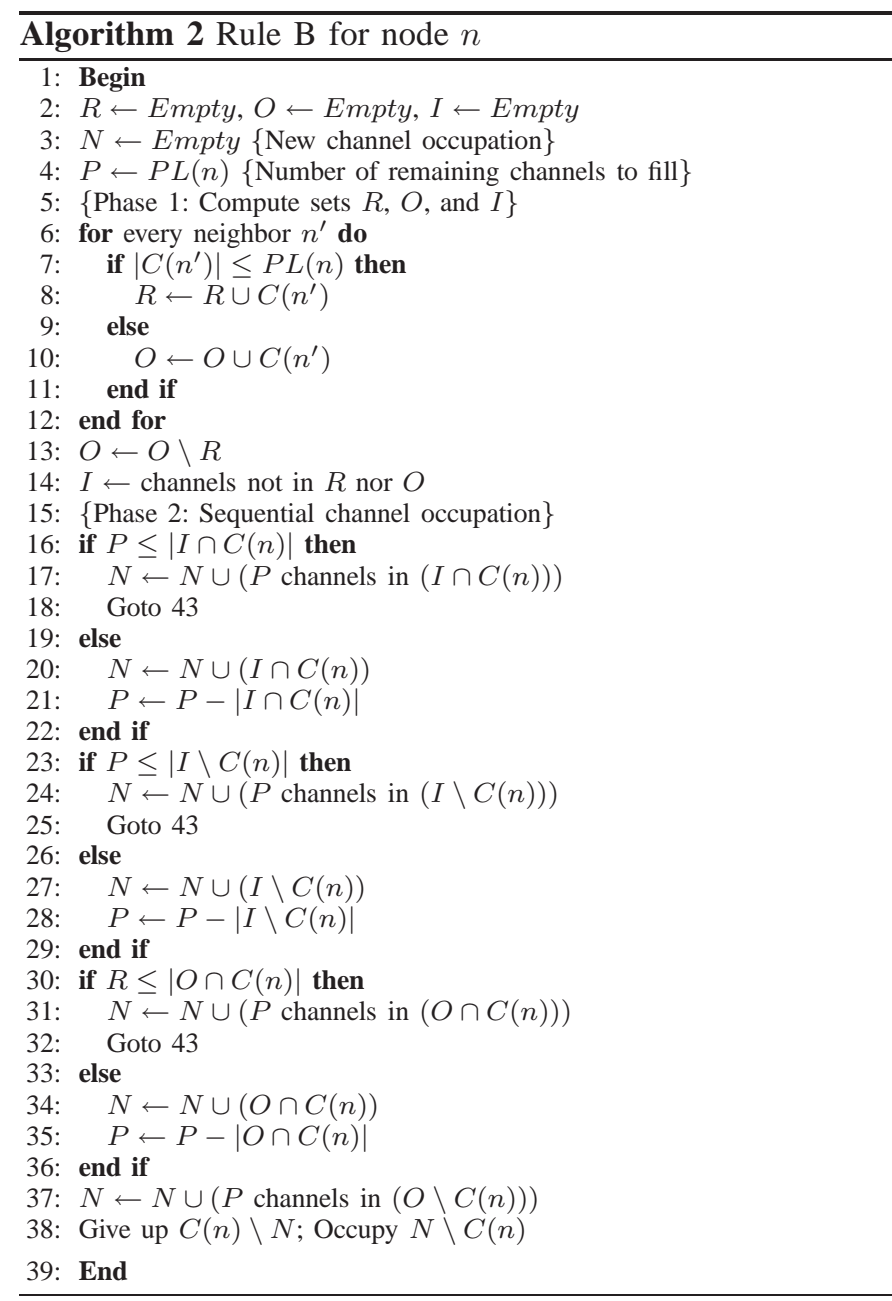

\section{B. Implementing Rule $D$ and $E$}

Essential to Rule D and E is the metric to determine the "best" channels. In Section V, we use the number of competing nodes as the channel quality indicator, assuming each node's traffic is backlogged. This metric can be inaccurate since in practice, nodes carry different and timevarying traffic load. Using IEEE 802.11 CSMA/CA devices, we propose to use busy time ratio (BTR) as the channel quality indicator [25]. Each node constantly monitors the available channels, and records the percentage of channel being busy and computes BTR. This new metric accounts for impact of node contentions, traffic heterogeneity, transmission failures and retransmissions. Nodes in close proximity can perform collaborative sensing to reduce complexity and energy consumption associated with channel monitoring/sensing [25]. This allows each node to obtain status of entire channel set by monitoring only a small fraction of the set. To avoid simultaneous channel adjustment at neighboring nodes, each node applies a random wait scheme described earlier before starting transmission on the new channels.

\section{EXPERIMENTAL RESULTS}

In this section, we conduct experimental simulations to quantify the performance of the rule based spectrum access.
We compare the proposed approach to those using explicit coordination and centralized optimization.

\section{A. Experiment Setup}

We assume a network of many WiFi and WiMAX access points. We assume each AP serves a large number of subscribers and have backlogged traffic. We use a simple binary interference model to construct the conflict graph - two nodes conflict if they are within distance of $D$. By default, we set $D$ to $100 \mathrm{~m}$. We use this assumption to simplify the conflict graph construction. However, it will not limit the scope of the proposed rule design. To examine the impact of network topology, we use both randomly generated topologies and measured AP deployment traces to validate our results.

- Random network. We place nodes randomly in an area.

- Clustered network. We simulate a hotspot scenario by deploying a set of nodes densely in a small area of the random network. We use this topology to examine the impact of conflict degree on the rule performance.

- Real network trace. We extract a set of actual AP deployments using data traces collected by Placelab. (http://www.placelab.org/).

We simulate both small and large networks. We use small networks to compare the performance of implicit coordination to that of centralized optimization. We use large networks to compare the performance of implicit coordination to that of explicit coordination. The real network trace contains 200 nodes located in a $200 \mathrm{~m}$ x $2000 \mathrm{~m}$ area. Hence, we construct random and clustered network in the same area with 200 nodes. For small networks, we deploy 20-40 nodes in an area of $1000 \mathrm{mx} 1000 \mathrm{~m}$, who contend for 20 channels. We compare the performance of Rule A, B, C, D and E based implicit coordination schemes to that of explicit coordination [6] (marked as CA-EC) and centralized assignment [20], [28] (marked as CA) schemes.

We design rules to maximize fairness utility, defined by Eq. (3). We evaluate the rule performance using both fairness and spectrum utilization ${ }^{2}$. For randomly generated topologies, we run the experiments over 500 topologies. For Rule C, D and $\mathrm{E}$, the system has multiple equilibriums with different spectrum usage. We record the average performance over many equilibriums. By default, we assume $\lambda=1.8$ for Rule D and $\mathrm{E}$ and set duty cycle to 1 . By default, we assume no primary nodes.

\section{B. Comparing Different Rules}

We start by examining the effectiveness of poverty line guided design by comparing Rules $\mathrm{A}$ to $\mathrm{B}, \mathrm{C}$, and $\mathrm{D}$ to $\mathrm{E}$. We assume a random network topology.

Rule A vs. B vs. C Figure 3 compares system utilities with Rule A, B and C. Clearly, Rule B and C significantly outperform Rule A. As the number of nodes per area increases, the system using Rule A is limited by the node with the worst conflict condition and the lowest poverty line. On the other

\footnotetext{
${ }^{2}$ The spectrum utilization is the sum of total node throughput $\sum_{n=0}^{N-1} R_{\mathbb{A}}(n)$.
} 

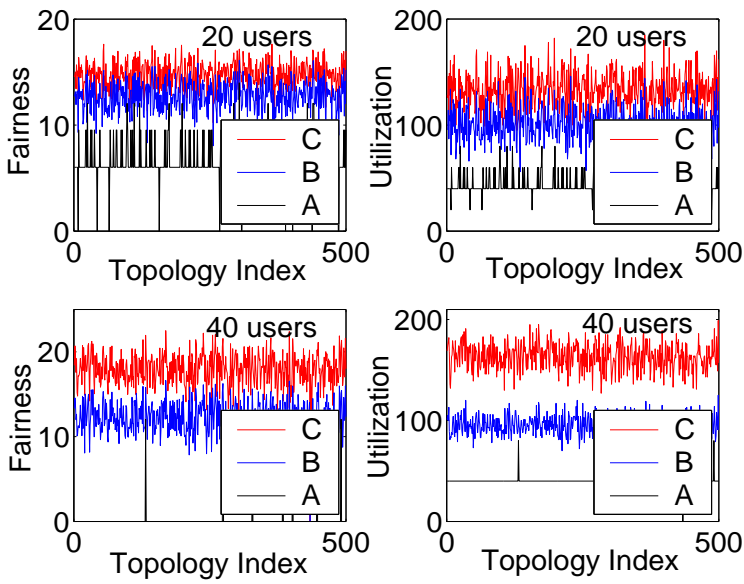

Fig. 3. Comparison of of Rule A and B in terms of the spectrum utilization (sum of total node throughput) and fairness (defined by Eq. (3)) assuming 20 channels and 20-40 nodes.
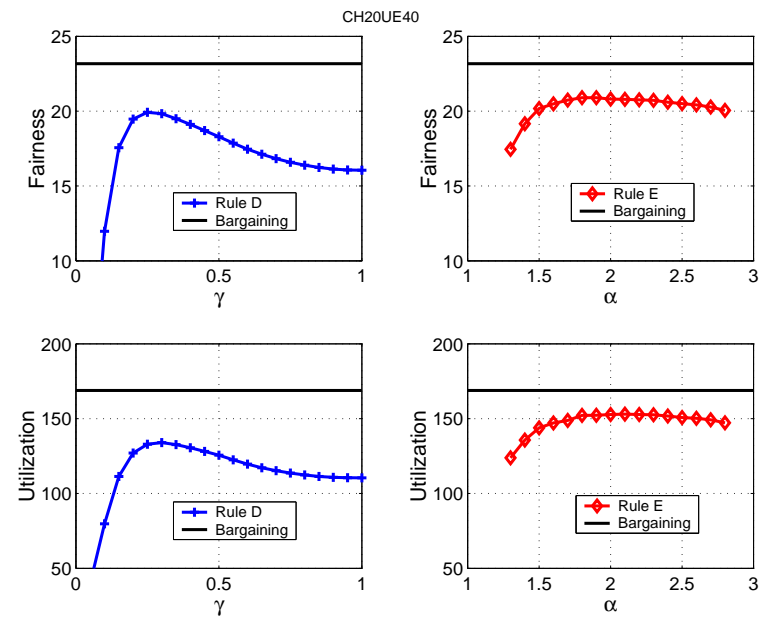

Fig. 4. Comparison of Rule D and $\mathrm{E}$ with the explicit coordination scheme. $\gamma=\Psi / M$, average results of 40 nodes sharing 20 channels.

hand, Rule B and C use local poverty line and adapt gracefully to the node density. Rule C outperforms Rule B by allowing some nodes to obtain more spectrum than their poverty line.

Rule D vs. E Figure 4 compares the performance of Rule $\mathrm{D}$ and $\mathrm{E}$ using two system utilities averaged over 500 random topologies under different $\gamma$ and $\alpha$ values. We see that Rule $\mathrm{D}$ is very sensitive to $\gamma-$ Small $\gamma$ leads to under-utilization of spectrum while large $\gamma$ (aggressive spectrum usage) results in excessive interference. On the other hand, Rule E is relatively insensitive to $\alpha$. Using $\alpha>1.5$, Rule E always outperforms Rule D. Hence, in the following, we will use $\alpha=1.8$ as the default setting of Rule E. We leave the derivation of the optimal $\alpha$ to a future study.

Complexity In Figure 5, we measure the complexity of different algorithms in terms of the total number of spectrum adjustments before the system reaches an equilibrium. We use a small network with 20 channels and 40 nodes. Compared to the explicit coordination approach, Rule $\mathrm{C}$ provides more than $60 \%$ reduction in complexity, and thus much faster adaptation to topology changes. Rule E offers similar number

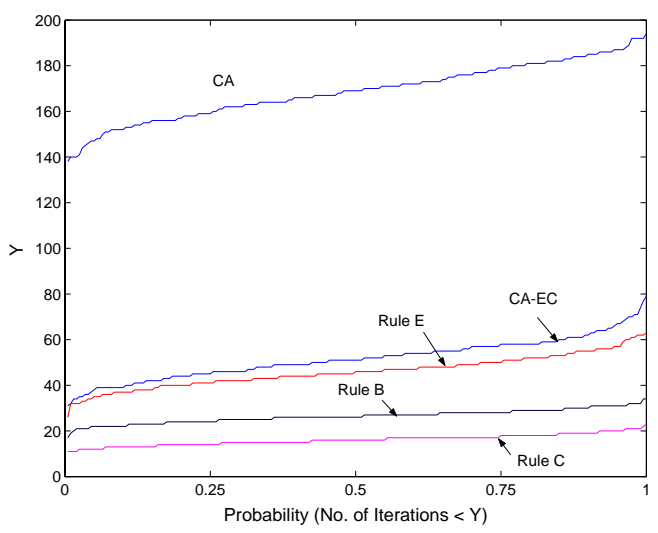

Fig. 5. Comparison of centralized (CA), explicit coordination (CA$\mathrm{EC}$ ), Rule B, C and $\mathrm{E}$ in term of complexity (the total number of spectrum adjustments), plotted as the cumulative distributive function of 500 topologies. We assume 20 channels shared by 40 access points.

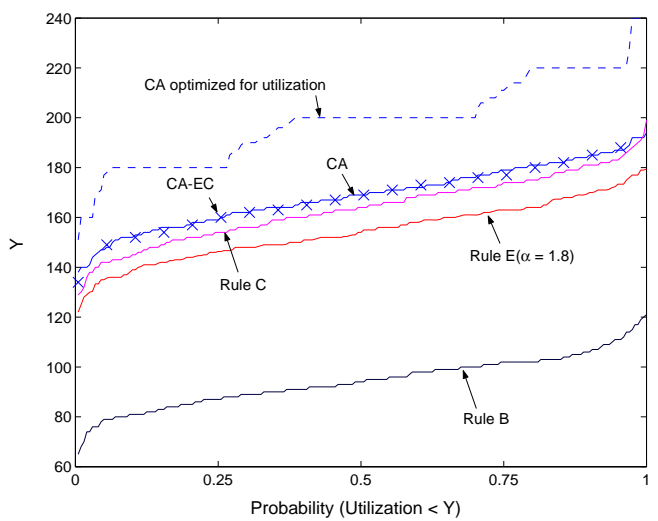

(a) Utilization

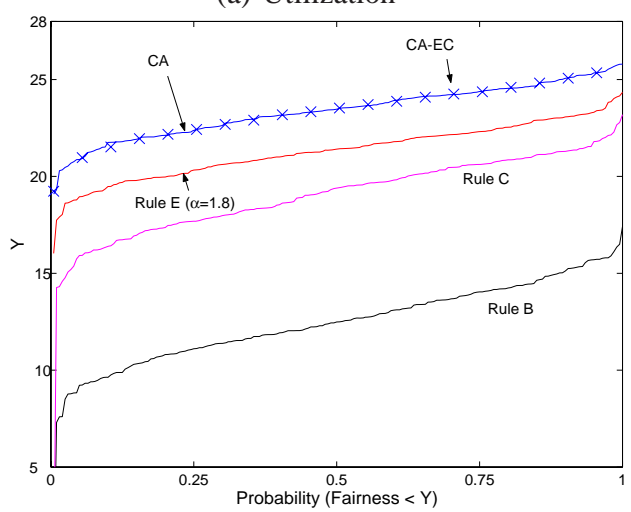

(b) Fairness

Fig. 6. Comparison of centralized (CA), explicit coordination (CA$\mathrm{EC})$, Rule B, C and E in term of system utilization and fairness utilities, plotted as the cumulative distributive function of 500 topologies. We assume 20 channels shared by 40 access points.

of adjustments as the explicit coordination approach.

\section{Comparing to Explicit Coordination}

In Figure 6 we compare Rule B, C and $\mathrm{E}$ to $\mathrm{CA}$ and CA-EC, using small random networks. As a reference, we also include the performance of centralized assignments that maximize utilization based utility. Similarly, there is a notice- 
able performance gap between rules and CA, CA-EC. This is as predicted because centralized optimization or distributed explicit coordination can improve system performance at the cost of higher complexity. However, we see that the proposed simple, implicit coordination leads to a graceful degradation in both utilization and fairness.

In Figure 5, we also compare the complexity of different approaches. For the collaboration based approaches (CA and CA-EC), a 4-way handshaking is required for each spectrum adjustment, so that the message overhead is 4 times the number of adjustments. For Rule C, each node broadcasts its spectrum usage to neighbors after the adjustment, resulting in one message per adjustment. In terms of coordination overhead, Rule C leads to $240 \%$ reduction compared to the explicit coordination approach. Because of its minor degradation in system utility and significant reduction in complexity, Rule $\mathrm{C}$ is a good low-complexity alternative to collaboration based approaches. For Rule E, if each node can measure channel utilization effectively, no message exchange is required. Given its low communication requirements, Rule $\mathrm{E}$ is a low-communication alternative.

\section{Impact of Network Topology}

Next, we examine the impact of network topology on node spectrum assignments, system utility and complexity. Figure 7 illustrates three sample topologies corresponding to large scale random, clustered and measured networks.

Node Spectrum Assignment. Figure 8 plots the number of channels assigned to each node using Rule C. We observe that a node $n$ 's spectrum usage is inversely proportional to its conflict degree $d(n)$, as predicted by the poverty line.

To further investigate this dependency, we plot in Figure 9 the amount of spectrum assigned at each node divided by its poverty line, as a function of the conflict degree $d(n)$. Clearly, there are two types of nodes: one has assignments very close to their poverty line, and another has assignments much higher than the poverty line. nodes of the first type in general have clique-like local conflict graph. They are located in dense areas where neighbors all conflict with each other. In this case, the poverty line is very tight. On the other hand, nodes of the second type has star-like local conflict topology, and their poverty line becomes loose.

Figure 10 shows the cumulative distribution function of the assignment using Rule $\mathrm{C}$ divided by its poverty line (RuleC/PL) for all three types of topologies. We see that almost $70 \%$ of nodes obtain spectrum assignment similar to their poverty line. Overall, we see that the poverty line provides a fairly reasonable estimation of node spectrum assignment and it is critical to incorporate poverty line into rule design.

Figure 10 also compares the performance of Rule $\mathrm{C}$ based implicit coordination scheme to CA-EC. We examine the cumulative distribution function of the assignment using explicit coordination divided by the one using Rule C (CA-EC/RuleC). All three topologies show that there are noticeable gaps at individual node's spectrum assignment. We notice that some nodes get less spectrum assignments using explicit coordination. This is because such assignments improve the proportional fair

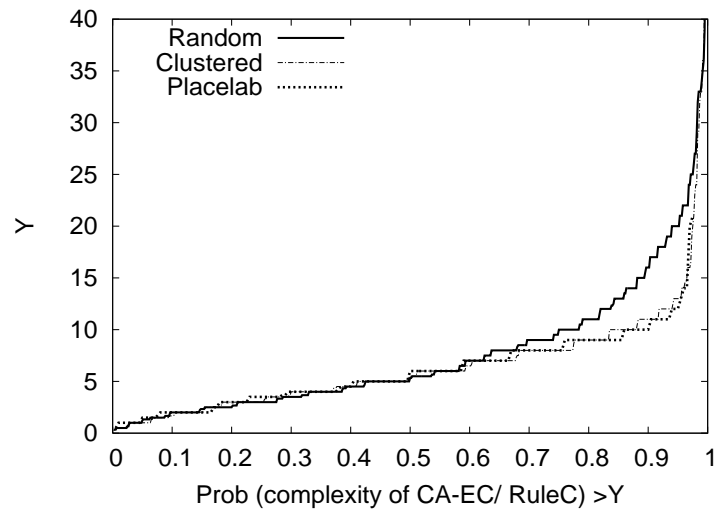

Fig. 13. The number of spectrum adjustments at each node using CAEC divided by those using Rule $\mathrm{C}$, for (left) random, (center) clustered and (right) Placelab networks, plotted as the cumulative distributive function over 200 nodes.

based utility function. However, we confirm both explicit and implicit coordination schemes always guarantee poverty line for all the nodes.

System Utility. Figure 11 compares the system fairness utility of Rule C and CA-EC for random, clustered and measured networks with 200 nodes. We vary the conflict distance $D$ from 20 to 200 meters. Without any explicit communication, rule $\mathrm{C}$ leads to a minor degradation compared to CA-EC. The difference increases with the conflict distance. Hence, the proposed implicit coordination is a good alterative to the explicit coordination approach under mild node deployments.

Complexity. Figure 12 plots the number of spectrum adjustments at each node for the system to reach an equilibrium, versus the conflict degree. Since our scheme always starts from an empty assignment, each node needs at least one adjustments. We see that most nodes need only 2-3 adjustments, although there are 100 channels.

Next, in Figure 13 we examine the number of spectrum adjustments required by CA-EC divided by those required by Rule $\mathrm{C}$. We see that Rule $\mathrm{C}$ can reduce coordination complexity by 4-10 times. We note that the complexity of CA-EC scales with the number of channels. Overall, we see that Rule $\mathrm{C}$ is highly favorable for networks with large number of channels.

\section{E. Impact of Primary/Legacy Devices}

We now examine spectrum usage in the presence of primary/legacy devices who own the spectrum. We randomly deploy a set of primary devices in the network, each occupying one channel. Each node obtains its spectrum availability and follows the rules to select channels. In Figure 14, we measure the fairness and utilization of allocations derived from Rules $\mathrm{C}$ and $\mathrm{E}$ and the explicit coordination approach (CAEC). The utilities are averaged over 100 randomly deployed primary nodes. We see that as primary devices increase their spectrum usage, the performance of both explicit and implicit coordination approaches degrade gracefully. 

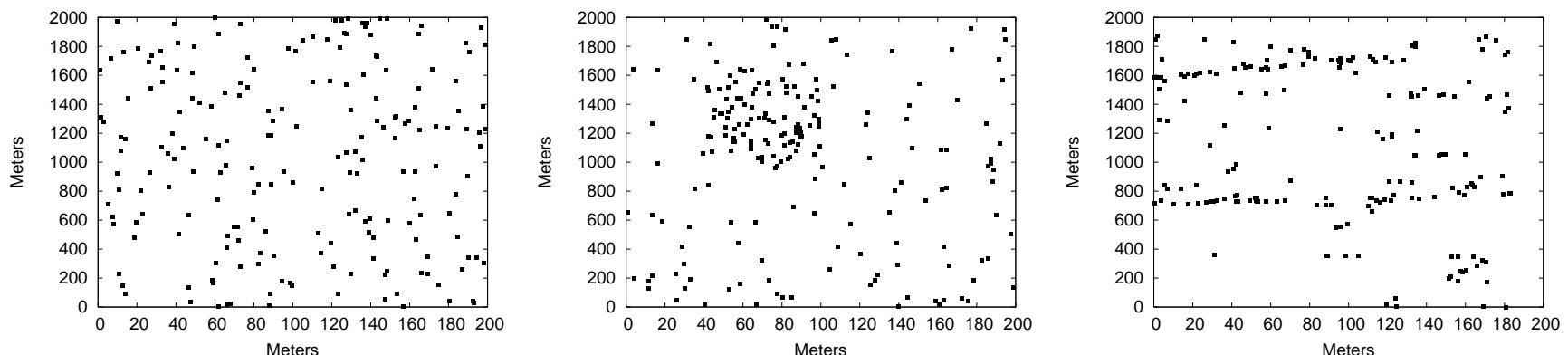

Fig. 7. Sample topology of (left) random network, (center) clustered network and (right) Placelab network.
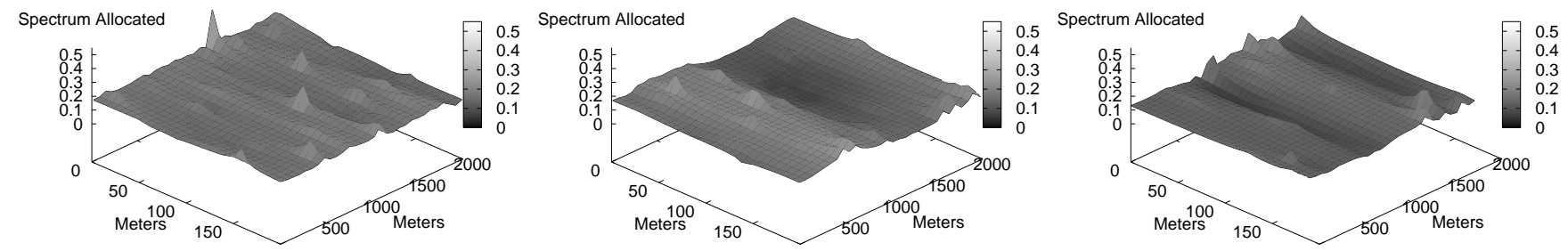

Fig. 8. The amount of spectrum allocated to each node using Rule $\mathrm{C}\left(R_{r u l e C}(n)\right)$ for (left) random network, (center) clustered network and (right) Placelab network shown in Figure 7.
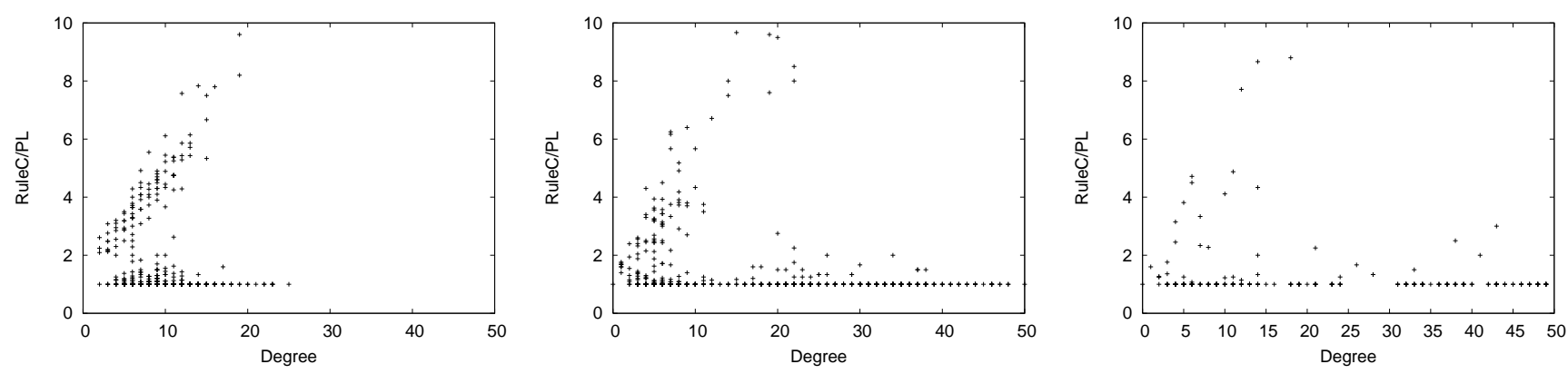

Fig. 9. The amount of spectrum assigned to each node using Rule C divided by its poverty line $\left(\frac{R_{r u l e C}(n)}{P L(n)}\right)$, versus node conflict degree $d(n)$ for (left) random, (center) clustered and (right) Placelab networks.

\section{DISCUSSION}

In this section, we discuss practical issues associated with our network model and problem definition.

Identifying channel availability and interference constraint. The proposed spectrum allocation system requires nodes (access points) to keep track of the channel availability matrix $\mathbb{L}$ and the interference constraint $\mathbb{C}$. We list three complementary mechanisms to obtain this information.

- Access points perform spectrum measurements to determine $\mathbb{L}$ and $\mathbb{C}$, using techniques like spectrum sensing [5], [7] and RSSI measurement-based probing [22].

- Access points can broadcast "hello" beacons [26] periodically and help peers construct the interference matrix.

- Clients associated with APs can assist the interference detection by sensing radio signals and provide feedback on findings of interfering access points [14]. This mecha- nism has been shown to help refine the interference map.

Extending to complex interference characterizations. In this paper, we use a binary matrix to model the pair-wise interference among access points. This model is widely used in existing works (e.g. [11], [14], [20]). We note that more realistic models are based on aggregated SNR measurements, referred to as the physical model [11]. However, under this model the corresponding channel allocation problem becomes significantly complex, and there are no existing analytical bounds on individual node's spectrum usage. We are currently investigating spectrum allocation schemes under this complex interference model, from which we seek to derive analytical bounds on node's spectrum usage and devise spectrum rules accordingly.

Finally, in this paper, we assume interference conditions are homogeneous across channels. Our model is a reasonable abstraction of the scenario where channels are divided 

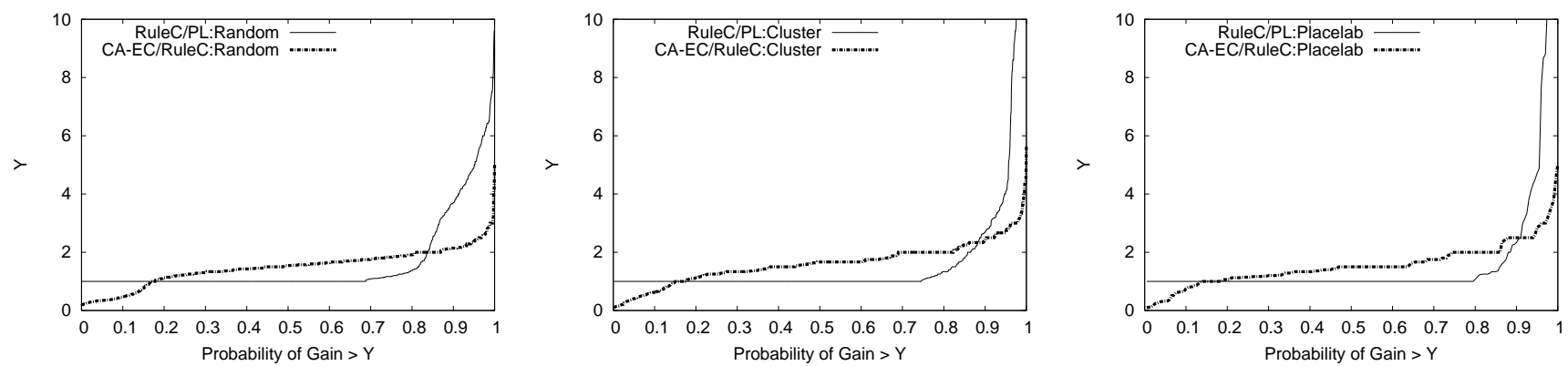

Fig. 10. Comparing the spectrum allocation per node using Rule C to poverty line: $\frac{R_{r u l e C}(n)}{P L(n)}$ (marked by RuleC/PL), using explicit coordination over those using Rule C: $\frac{R_{C A-E C}(n)}{R_{r u l e C}(n)}$ (marked by CA-EC/RuleC) for (left) random,(center) clustered and (right) Placelab networks, plotted as the cumulative distributive function over 200 nodes.
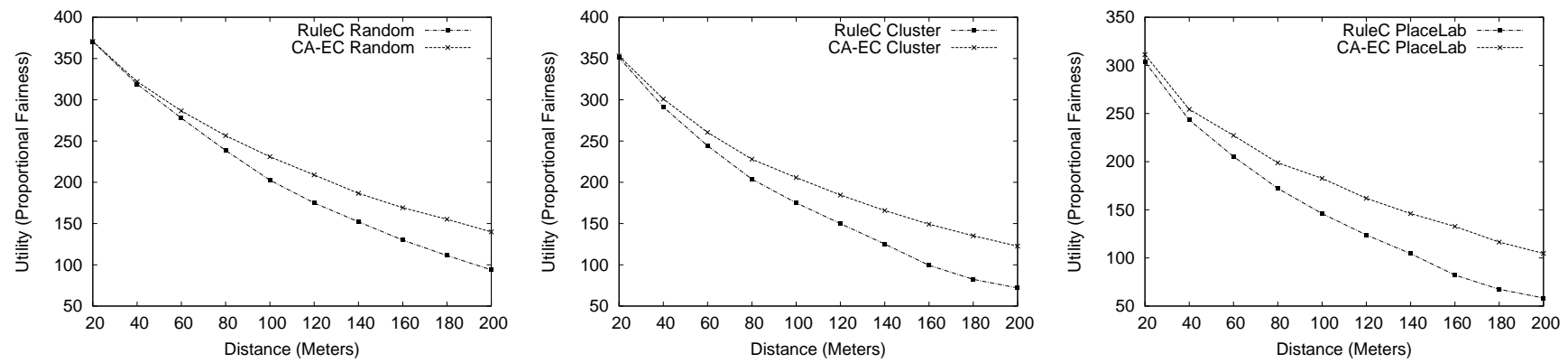

Fig. 11. The system fairness utility of Rule $\mathrm{C}$ and CA-EC versus the conflict distance $D$, for (left) random,(center) clustered and (right) Placelab networks.
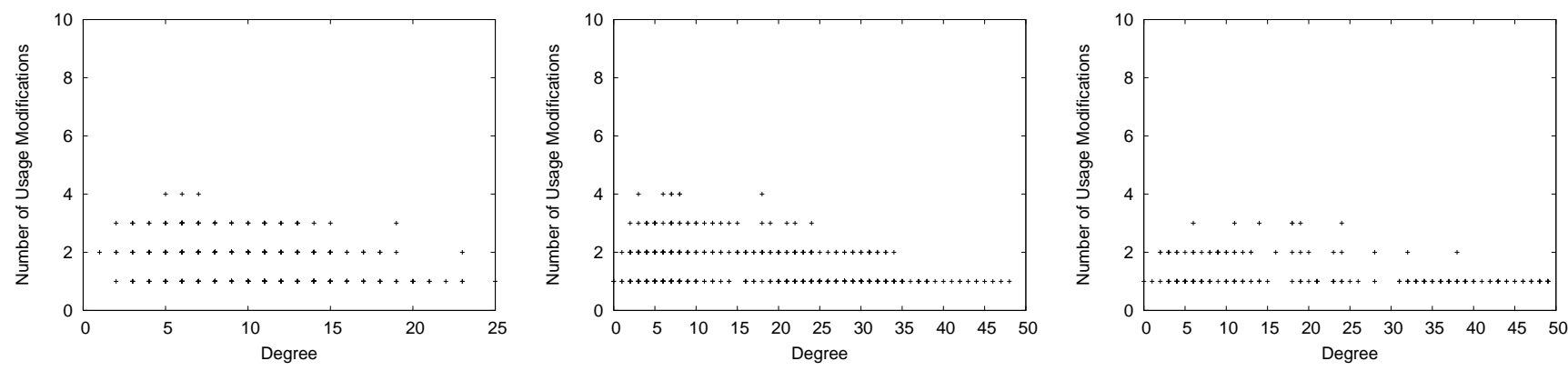

Fig. 12. The number of spectrum adjustments at each node versus the node conflict degree $d(n)$, for (left) random, (center) clustered and (right) Placelab networks.

from a single band pool and have frequencies close to each other. When interference conditions are heterogeneous across channels, we can use an aggregated measure of interference across channels to define the interference constraint and apply the proposed solution, i.e. any two nodes conflict if they conflict on at lease one channel. However, this reduction is overly conservative and can degrade spectrum utilization. An alternative is to make the interference constraint channeldependent, i.e. $c_{n, k, m}$. Our prior work in [20] has developed centralized approaches to optimize spectrum allocations under these channel-dependent constraints. We are currently investigating analytical bounds on individual node spectrum usage and applying these bounds to design rules for the proposed distributed implicit coordination approach.

\section{CONCLUSION}

We propose a distributed spectrum management scheme using implicit coordination. Nodes adjust spectrum usage independently while complying with spectrum rules, leading to significantly less communication and computational overhead. We propose five rules that tradeoff performance with implementation and communication complexity. We show that rules guided by a lower bound on nodes' spectrum usage (poverty line) provide superior performance. Experimental results show that rule-based approaches perform slightly worse than the previously proposed collaborative approaches, but have much lower complexity and communication overhead.

\section{REFERENCES}

[1] Business 2.0 magazine: Get ready for googlenet, 2005. 


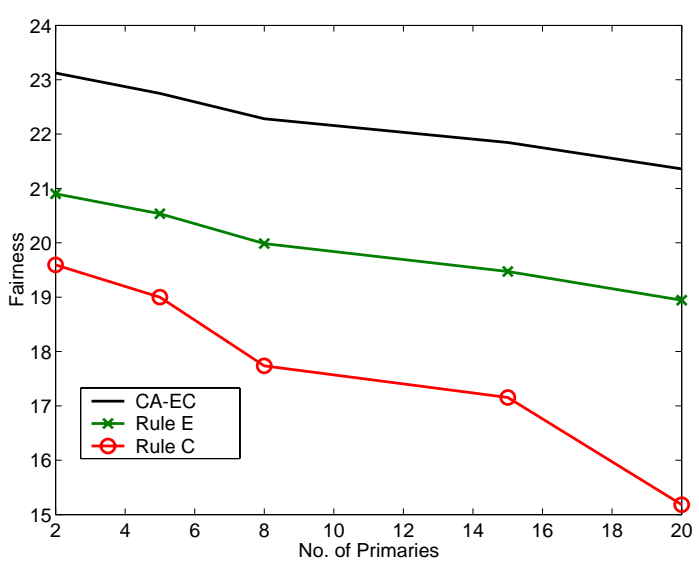

(a) Fairness

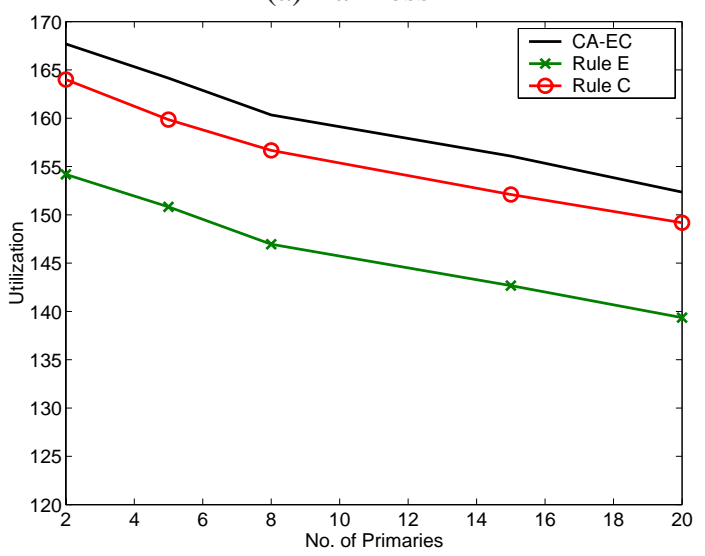

(b) Utilization

Fig. 14. Utilization and Fairness comparison of different rules assuming 20 channels, 40 secondary nodes and 20 primary nodes.

[2] Akella, A., Judd, G., Seshan, S., and Steenkiste, P. Selfmanagement in chaotic wireless deployments. In Proc. of MobiCom (August 2005).

[3] BRUAldi, R. A. Introductory Combinatorics, 3rd ed. Prentice Hall, Englewood Cli s, N.J., 1999.

[4] Buddhikot, M. M., Kolody, P., Miller, S., Ryan, K., And EVANS, J. DIMSUMNet: New directions inwireless networking using coordinated dynamic spectrum access. In Proc. of IEEE WoWMoM (June 2005).

[5] Cabric, D., Mishra, S. M., And Brodersen, R. W. Implementation issues in spectrum sensing for cognitive radios. In Proceedings of Asilomar conference on signals, systems and computers (2004).

[6] CAO, L., AND Zheng, H. Spectrum allocation in ad hoc networks via local bargaining. In Proc. of SECON (September 2005).

[7] G. Ganesan, Y. L. Implementation issues in spectrum sensing for cognitive radios. In Proc. IEEE DySPAN 2005 (November 2005).

[8] HAYkin, S. Cognitive radio: Brain-empowered wireless communications. IEEE Journal on Selected Areas in Communications 23, 2 (Feburary 2005), 201-220.

[9] Heusse, M., Rousseau, F., Berger-Sabbatel, G., and Duda, A. Performance anomaly of 802.11 b. In Proc. of INFOCOM (March 2003).

[10] Ileri, O., Samardzija, D., And Mandayam, N. B. Demand responsive pricing and competitive spectrum allocation via spectrum server. In Proc. of IEEE DYSPAN (November 2005).

[11] Jain, K., Padhye, J., Padmanabhan, V., And Qiu, L. Impact of interference on multi-hop wireless network performance. In Mobicom 2003.

[12] L. Jiang, S. C. L. Proportional fairness in wireless lans and ad hoc networks. In Proc. IEEE WCNC (2005).

[13] McHenry, M. Spectrum white space measurements. New America Foundation Broadband Forum (June 2003).

[14] Mishra, A., ET AL. Distributed channel management in uncoordinated wireless environments. In Proc. of MobiCom (September 2006).
[15] Mitola III, J., And Maguire JR., G. Q. Cognitive radio: making software radios more personal. IEEE Personal Communications 6, 4 (August 1999), 13-18.

[16] Nandagopal, T., T.Kim, X.Gao, and Bharghavan, V. Achieving MAC layer fairness in wireless packet networks. In Proc. of MobiCom (August 2000).

[17] NeEl, J., AND ReEd, J. Performance of distributed dynamic frequency selection schemes for interference reducing networks. In Proc. of Milcom (October 2006).

[18] Neel, J., ReED, J., AND Gilles, R. The role of game theory in the analysis of software radio networks. In Proc. Software Defined Radio Forum Technical Conference and Product Exhibition (SDR 02) (San Diego, CA, USA, November 2002), vol. 2, pp. pp. NP-3-02.

[19] NiE, N., AND ComaniciU, C. Adaptive channel allocation spectrum etiquette for cognitive radio networks. In Proc. of IEEE DySPAN (November 2005).

[20] Peng, C., Zheng, H., And Zhao, B. Y. Utilization and fairness in spectrum assignemnt for opportunistic spectrum access. Mobile Networks and Applications (MONET) 11 (May 2006), 555-576.

[21] Raman, C., Yates, R. D., and Mandayam, N. B. Scheduling variable rate links via a spectrum server. In Proc. of IEEE DySPAN (November 2005)

[22] Reis, C., Mahajan, R., Rodrig, M., Wetherall, D., and ZaHORJAN, J. Measurement-based models of delivery and interference in static wireless networks. In Proc. of ACM SigComm (2006).

[23] Steenstrup, M. Opportunistic use of radio- frequency spectrum: a network perspective. In Proc. of IEEE DySPAN (November 2005).

[24] Xing, Y., Chandramouli, R., Mangold, S., And Shankar, S. Dynamic spectrum access in open spectrum wireless networks. IEEE Journal on Selected Areas in Communications 24, 3 (March 2006), 626637.

[25] Yang, G., Zheng, H., ZhaO, J., AND LI, V. Adaptive channel selection through collaborative sensing. In Proc. of ICC (June 2006).

[26] ZhaO, J., Zheng, H., AND YANG, G. Distributed coordination in dynamic spectrum allocation networks. In Proc. of IEEE DySPAN (November 2005).

[27] Zheng, H., And CaO, L. Device-centric spectrum management. In Proc. of IEEE DySPAN (November 2005).

[28] Zheng, H., AND Peng, C. Collaboration and fairness in opportunistic spectrum access. In Proc. of ICC (June 2005).

\section{A. Proof of Theorem 1}

A node $i$ observes its neighbors' spectrum usage when selecting channels. The total number of channels occupied by its neighbors is at most $O(i)=$ $\Omega \cdot d(i)$. Obviously, $\Omega \leq L(i)-O(i)$. Hence, node $i$ can always find at least $\Omega$ idle channels that will not lead to any conflict.

\section{B. Proof of Theorem 2}

\section{B.1 Proof for Rule B}

Definition 1: We define a node $n$ as "qualified" if $|C(n)|=P L(n)$, and $n$ does not conflict with any node who uses less number of channels than $P L(n)$. Otherwise, the node is "disqualified".

We start with a lemma that describes how the status of a node switches by running Rule B.

Lemma 1: After a node $n$ performs a spectrum adjustment based on rule $B$, it becomes "qualified".

Proof: It is equivalent to prove that the sets $O$ and $I$ are large enough to fill $P L(n)$ channels, i.e. $|O|+|I| \geq P L(n)$ in Algorithm 2 .

There are at most $d(n)$ neighbors of $n$ whose channel usage is less than $P L(n)$, denoted as $n_{1}, n_{2}, \cdots, n_{i}, i \leq d(n)$. Hence,

$$
|B|=\left|\bigcup_{s=1}^{i} C\left(n_{s}\right)\right| \leq \sum_{s=1}^{i}\left|C\left(n_{s}\right)\right| \leq d(n) \times P L(n) \leq \frac{d(n)}{d(n)+1} L(n) .
$$

Therefore, $|O|+|I|=L(n)-|B| \geq P L(n)$.

The following lemma describes how a node affects the status of its neighbors by running Rule B.

Lemma 2: When a node $n$ adjusts its spectrum usage and converts any of its neighbors, i.e. node $n^{\prime}$ from "qualified" to "disqualified", then $P L(n)<$ $P L\left(n^{\prime}\right)$. Moreover, $n$ must be "disqualified" before its spectrum adjustment.

Proof: Since $n^{\prime}$ is "qualified" before $n$ 's adjustment, $n^{\prime}$ 's channel usage before adjustment is $\left|C\left(n^{\prime}\right)\right|=P L\left(n^{\prime}\right)$. If $P L(n)>P L\left(n^{\prime}\right)$, then after 
the spectrum adjustment, $n$ 's channel usage will not conflict with that of $n^{\prime}$, so $n^{\prime}$ won't change from "qualified" to "disqualified".

To see $n$ must be "disqualified" before the spectrum adjustment, assume on the contrary that it is "qualified" before the spectrum adjustment. According to the rule, the only motivation that $n$ modifies its channel usage is there are more idle channels (i.e. set $I \backslash C(n)$ is not empty) so $n$ seizes them and gives up the same amount of channels under conflict (i.e. in set $O \cap C(n)$ ). This modification does not alter $|C(n)|$ and does not create new conflicts, so $n^{\prime}$ won't change from "qualified" to "disqualified".

From lemma 2, node $n$ can only convert neighbors with higher Poverty Line from "qualified" to "disqualified", and a "qualified" node won't change any of its neighbors to "disqualified".

Lemma 3: For a given system with $N$ nodes and any initial channel assignment, after at most an expected number of $O\left(N^{2}\right)$ spectrum adjustments, all the nodes will become "qualified".

Proof: From lemma 2, if spectrum adjustment is carried out in a "structured" format such that "disqualified" nodes with lowest poverty line among its neighbors have the highest priority in adjusting spectrum, then each "qualified" node will never become a "disqualified" node. As a result, the total number of spectrum adjustment is at most $N$ for all nodes to become "qualified".

However, if the order of spectrum adjustment is random, then a "disqualified" node with the lowest poverty line among its neighbors will wait for an expected number of at most $N$ other adjustments to get the opportunity. This is mainly due to at each adjustment slot, the number of nodes who needs to adjust spectrum is bounded by $N$. Therefore the number of total spectrum adjustments until the system reaches an equilibrium is bounded by $O\left(N^{2}\right)$.

Now, we can use the above lemmas to prove theorem 2 for Rule B.

Proof of Theorem 2 for Rule B:

By lemma 3, after at most an expected number of $O\left(N^{2}\right)$ channel modifications all nodes are "qualified".

According to definition 1, when all nodes are "qualified", for every $n, \mathrm{n}$ is on its Poverty Line, and for every neighbor $n^{\prime}$ s.t. $P L(n) \geq\left|C\left(n^{\prime}\right)\right|=$ $P L\left(n^{\prime}\right), n$ doesn't conflict with $n^{\prime}$. This implies the global assignment is conflict-free. It is just the status of equilibrium.

\section{B.2 Proof for Rule $C$}

Definition 2: We define a node $n$ as "qualified" if one of the following conditions is satisfied.

1) $|C(n)|>P L(n)$, and the $P L(n)$ channels with the smallest channel IDs occupied by $n$ (hereby referred to as upper channels) do not generate conflict with any neighbor of $n$.

2) $|C(n)|=P L(n)$, and for every neighbor $n^{\prime}, n$ doesn't conflict with $n^{\prime}$ on the upper $\min \left(P L(n),\left|C\left(n^{\prime}\right)\right|\right)$ channels occupied by $n^{\prime}$.

Otherwise, the node is "disqualified".

We start with a lemma that describes how the status of a node switches by running Rule C.

Lemma 4: , After $n$ performs a channel modification based on rule $C, n$ becomes "qualified".

Proof: If $|I| \geq P L(n)$, then after applying Rule $\mathrm{C} n$ will occupy all idle channels and won't conflict with any neighbor, so the qualification is trivial. Otherwise if $|I|=P L(n)$ because Rule $\mathrm{C}$ is reserving the upper $\min \left(P L(n),\left|C\left(n^{\prime}\right)\right|\right)$ channels for each neighbor, the only thing remains to prove is sets $O$ and $I$ are enough for providing PL(n) channels, i.e. $|O|+|I| \geq$ $P L(n)$ in algorithm 1 .

There are at most $d(n)$ neighbors of $n$. Based on Rule C, node $n$ reserves at most $P L(n)$ channels into set $B$ for each neighbor. Therefore

$$
|B| \leq d(n) \times P L(n) \leq \frac{d(n)}{d(n)+1} L(n)
$$

Therefore, $|O|+|I|=L(n)-|B| \geq P L(n)$.

The following lemma describes how a node affects the status of its neighbors by running Rule $\mathrm{C}$.

Lemma 5: When a node $n$ adjusts its spectrum and converts any of its neighbors, i.e. node $n^{\prime}$ from "qualified" to "disqualified", then $P L(n)<$ $P L\left(n^{\prime}\right)$. In addition, $n$ is "disqualified" before its spectrum adjustment.

Proof: We first assume that $P L(n) \geq P L\left(n^{\prime}\right)$. Note $n^{\prime}$ is "qualified" before the spectrum adjustment. Therefore, there are two possible cases.

- If $\left|C\left(n^{\prime}\right)\right|>P L\left(n^{\prime}\right)$, then according to Rule $\mathrm{C} n$ will avoid conflict with the upper $P L(n)\left(\geq P L\left(n^{\prime}\right)\right)$ channels thus $n^{\prime}$ is still "qualified".
- If $\left|C\left(n^{\prime}\right)\right|=P L\left(n^{\prime}\right)$, then $P L(n) \geq\left|C\left(n^{\prime}\right)\right|$. Then according to the rule, after the modification $n$ won't conflict with $n^{\prime}$, so $n^{\prime}$ won't change from "qualified" to "disqualified".

Therefore, $P L(n) \geq P L\left(n^{\prime}\right)$ is impossible, and we have proved $P L(n)<P L\left(n^{\prime}\right)$

Next, we need to prove that $n$ must be "disqualified" before the spectrum adjustment. Let's first assume that $n$ is "qualified" before the adjustment. From definition 2, $n$ 's spectrum usage can be divided into the following two cases.

- If $|C(n)|>P L(n)$, and the upper $P L(n)$ channels occupied by $n$ do not generate any conflict with any neighbor. During the spectrum adjustment, $n$ will simply exit from the conflicted channels since the number of idle channels is enough for $n$ to reach its Poverty Line. After that $n$ won't conflict with its neighbor $n^{\prime}$, which means that $n^{\prime}$ won't become "disqualified".

- If $|C(n)|=P L(n)$. Since $n$ is "qualified", the conflicting set $B$ is disjoint with $C(n)$. According to the rule, the only motivation that $n$ modifies its channel usage is there are more idle channels (i.e. set $I \backslash C(n) \neq \emptyset$ ). During spectrum adjustment, $n$ seizes a number of idle channels and exits from the same amount of channels that are conflicting (i.e. channels in the set $O \cap C(n)$ ). This modification does not alter $|C(n)|$ and does not create new conflicts. Now $n^{\prime}$ s spectrum usage can be divided into two cases:

If $\left|C\left(n^{\prime}\right)\right|>P L\left(n^{\prime}\right)$ and the upper $P L\left(n^{\prime}\right)$ channels of $n^{\prime}$ are free of conflict, then after $n$ modifies its channel usage those channels are still free of conflict thus $n^{\prime}$ is still "qualified"; If $\left|C\left(n^{\prime}\right)\right|=P L\left(n^{\prime}\right)$, because $P L\left(n^{\prime}\right)>P L(n)$ and $P L(n)=|C(n)|$, it is straightforward that $\left|C\left(n^{\prime}\right)\right|>|C(n)|$. Because $n^{\prime}$ is "qualified" before the modification of $n, n^{\prime}$ doesn't conflict with $n$. This means that after $n$ 's spectrum adjustment, $n^{\prime}$ and $n$ do not conflict with each other. Hence, $n^{\prime}$ is still "qualified".

In conclusion, if $n$ is "qualified", then its channel modification won't transfer $n^{\prime}$ into "disqualified".

From lemma 5, node $n$ can only convert neighbors with higher Poverty Line from "qualified" to "disqualified", and a "qualified" node won't change any of its neighbors to "disqualified".

Lemma 6: For a given system with an arbitrary initial assignment, through at most an expected number of $O\left(N^{2}\right)$ channel modifications all nodes will become "qualified".

Proof: From lemma 5, the proof is quite similar as the proof for lemma

Now, we can use the above lemmas to prove theorem 2 for Rule C.

By lemma 6, after at most an expected number of $O\left(N^{2}\right)$ channel modifications all nodes are "qualified". At this point, some "qualified" nodes can still modify their channel usage. We hereby refer to a "qualified" node whose spectrum usage is above its poverty line (i.e. $|C(n)|>P L(n)$, as the first clause in definition 2) as type I node, and a node whose spectrum usage is equal to its poverty line(i.e. the second clause in definition 2) as type II nodes.

When all nodes are "qualified", there may still exist some conflicts. However two type II nodes are not possible to conflict: the one with higher or equal Poverty Line will avoid conflict with the other. So conflict only exists between one Type I node and a Type II node, or between two Type I nodes. Type I nodes modify their spectrum usage by exiting from channels that conflict with neighbors, so their modification eliminates the conflicts with their neighbors. So after at most $O\left(N^{2}\right)$ expected iterations all type I nodes modified their channel usage and the system is conflict-free.

When spectrum usage in a system becomes conflict-free and all nodes are "qualified", the only spectrum adjustment at a node is to seize idle channels. The number of this spectrum adjustment is at most $N$ before the system enters an equilibrium. Therefore, the expected number of spectrum adjustment for a system with $N$ nodes to reach an equilibrium is at most $O\left(N^{2}\right)$.

\section{Proof of Theorem 3}

We start the proof by defining a conflict graph $G=\{U, E\}$, where $U$ represents the list of vertices (the secondary nodes), $(|U|=N)$, and $E$ represents the edges between vertices. An edge exists between two vertices if the corresponding nodes conflict with each other if using the same channel. $\Lambda=|E|$ represents the number of edges in the conflict graph. It is obvious that the number of edges is maximized when the network is all connected, where $\Lambda=N(N-1) / 2$. Let $A$ characterize the network's spectrum usage, 
i.e. the channel(s) used by each secondary node/vertex. We now define the conflict factor of $A$ as

$$
C F(A)=\sum_{m=0}^{M-1} C F_{m}(A)
$$

where $C F_{m}(A)$ is the number of the pair of conflicting nodes who are using channel $m$. Following Rules D and E, a node $n$ switches from channel $m$ to $m^{\prime}$ only if it can gain more throughput from $m^{\prime}$ than from $m$. Based on the interference model, the number of nodes conflicting with $n$ on channel $m^{\prime}$ is smaller than that on channel $m$. Therefore, we can derive the following.

$$
\begin{array}{r}
C F_{m^{\prime}}\left(A^{\prime}\right)-C F_{m^{\prime}}(A)<C F_{m}(A)-C F_{m}\left(A^{\prime}\right) \Rightarrow \\
C F_{m^{\prime}}\left(A^{\prime}\right)+C F_{m}\left(A^{\prime}\right)<C F_{m}(A)+C F_{m^{\prime}}(A) .
\end{array}
$$

Since the switch will only impact on the values of $C F_{m}($.$) and C F_{m^{\prime}}($.$) ,$ it is obvious that $C F\left(A^{\prime}\right)<C F(A)$. Hence, $C F$ strictly decreases each time a node modifies its channel usage. $C F(A) \leq|E| \times M$ and $C F \in \mathcal{Z}$, the switch will end after at most $|E| \times M$ iterations, and the system will reach an equilibrium.

\section{Proof of Theorem 4}

Proof for $\Psi=1$ : By Pigeonhole Principle [3], there must exist a channel where a node $n$ observes less or equal than $\left\lfloor\frac{d(n)}{M}\right\rfloor$ neighbors. At an equilibrium, each node selfishly selects the best channel for itself, and conflicts with at most $\left\lfloor\frac{d(n)}{M}\right\rfloor$ neighbors.

Proof for $1<\Psi<M$ : Assuming that a node $n$ is assigned with $\Psi$ channels. By an inductive argument using Pigeonhole Principle, there must exist $\Psi$ channels, denoted $c_{1}, c_{2}, \cdots, c_{\Psi}$, such that

$$
d_{c_{1}}(n)+d_{c_{2}}(n)+\cdots+d_{c_{\Psi}}(n) \leq \Psi \times\left(\left\lfloor\frac{\Psi \times d(n)}{M}\right\rfloor+1\right) .
$$

Here we use $d_{c}(n)$ to denote the number of neighbors of $n$ who are occupying channel $c$. Now if node $n$ switches to these $\Psi$ channels, its utility will be

$$
\begin{aligned}
& \frac{1}{\lambda}\left(\frac{1}{d_{c_{1}}(n)+1}+\frac{1}{d_{c_{2}}(n)+1}+\cdots+\frac{1}{d_{c_{\Psi}}(n)+1}\right) \\
& \geq \frac{1}{\lambda} \frac{\Psi}{\left(\left(d_{c_{1}}(n)+1\right)\left(d_{c_{2}}(n)+1\right) \cdots\left(d_{c_{\Psi}}(n)+1\right)\right)^{\frac{1}{\Psi}}} \\
& \geq \frac{1}{\lambda} \frac{\Psi}{\left(\frac{\left(d_{c_{1}}(n)+1\right)+\left(d_{c_{2}}(n)+1\right)+\cdots+\left(d_{c_{\Psi}}(n)+1\right)}{\Psi}\right)^{\Psi \frac{1}{\Psi}}} \\
& =\frac{1}{\lambda} \frac{\Psi^{2}}{d_{c_{1}}(n)+1+d_{c_{2}}(n)+1+\cdots+d_{c_{\Psi}}(n)+1} \\
& \geq \frac{1}{\lambda} \frac{\Psi}{\Psi\left(\left\lfloor\frac{\Psi d(n)}{M}\right\rfloor+1\right)+m} \\
& =\frac{\Psi}{\lambda\left\{\left\lfloor\frac{\Psi d(n)}{M}\right\rfloor+2\right\}} .
\end{aligned}
$$

Proof for $\Psi=M$ : Straightforward following the interference model. For each node, there are $d(n)+1$ nodes competing for all $M$ channels. 